\title{
Face Recognition Using Chain Codes
}

\author{
Nazmeen B. Boodoo-Jahangeer, Sunilduth Baichoo
}

Department of Computer Science, University of Mauritius, Reduit, Mauritius.

Email: nazmeen182@yahoo.com

Received May, 2013.

\begin{abstract}
Face recognition is an active area of biometrics. This study investigates the use of Chain Codes as features for recognition purpose. Firstly a segmentation method, based on skin color model was applied, followed by contour detection, then the chain codes of the contours were determined. The first difference of chain codes were calculated since the latter is invariant to rotation. The features were calculated and stored in a matrix. Experiments were performed using the University of Essex Face database, and results show a recognition rate of $95 \%$ with this method, when compared with Principal Components Analysis (PCA) giving $87.5 \%$ recognition rate.
\end{abstract}

Keywords: Face Recognition; Biometrics; Chain Codes; PCA

\section{Introduction}

This study investigates the use of chain codes as features for face recognition. Features representing images are termed as descriptors. For robust recognition, descriptors should not be sensitive to variations like: Size Change, Translation and Rotation.

Common image descriptors include Area, Perimeter, Compactness and Eccentricity. However these descriptors are sensitive to variations. Such descriptors therefore cannot be used as reliable features to represent objects. Other object representations include Chain codes, Polygonal Approximation, Signatures, Convex Hull, Fourier Descriptor, Texture Content and Moments. The use of chain codes have not been explored enough in literature.

In this work, the use of chain codes as descriptor has been investigated. Face images have been pre-processed to find the contours. The chain codes of the contours are used as features for recognition.

\section{Background Study}

\subsection{Face Recognition}

Research in automatic face recognition dates back to the 1960's [1]. A survey of face recognition techniques has been given by Zhao, W. et al., [2]. In general, face recognition techniques can be divided into two groups based on the face representation they use:

(a) Appearance-based, which uses holistic texture features and is applied to either whole-face or specific regions in a face image; (b) Feature-based, which uses geometric facial features (mouth, eyes, brows, cheeks etc.) and geometric relationships between them.

\subsection{Chain Codes}

Chain codes are one of the techniques that can recognize characters and digits successfully. According to Seul et al [3], this technique possesses several advantages. Firstly, the chain codes are a compact representation of a binary object. Secondly, it is easier to compare objects using chain codes as they are a translation invariant representation of a binary object. Another advantage is that chain code can be used to compute any shape feature as it is a complete representation of an object or curve. Chain codes provide lossless compression and preserve all topological and morphological information which will be useful in the analysis of line patterns in terms of speed and effectiveness [4].

Jiang et al. (2009) made use of Chain codes to extract features of fingerprint. The fingerprint image captured was enhanced and binarized, the ridges of fingerprint image were thinned, then freeman chain code was derived to describe the extracted fingerprint ridge, matching based on fingerprint ridge contour. This method has proved to be the translation invariant. No results were reported by the authors

\section{Methodology}

In this section, the database used, the pre-processing techniques and the procedures followed are explained. 


\subsection{Pre-Processing}

\section{Method 1: Canny Edge Detection}

The original image is converted to grayscale and the canny edge detection is applied to the image. Figure 1 shows the results.

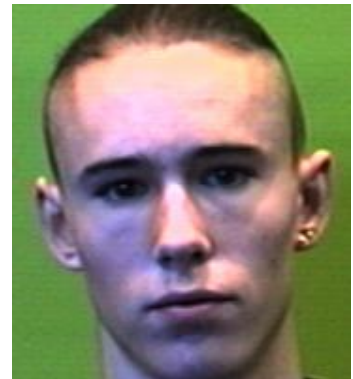

(a)

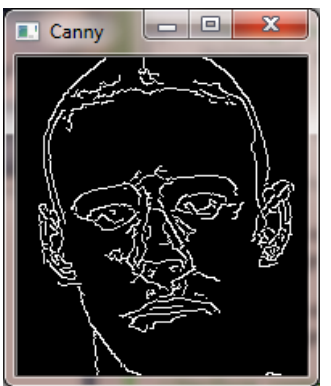

(b)
Figure 1. (a) Original Image, (b) Image Edges.

\section{Method 2: Skin Color Model}

Several skin color detection techniques have been used in literature [6,7]. A skin color model is applied for detecting the face and extracting the background. The method makes use of the $\mathrm{YC} \mathrm{rCb}$ color space [8]. $\mathrm{YCrCb}$ is an encoded nonlinear RGB signal, commonly used by European television studios and for image compression work. Color is represented by luma (which is luminance, computed from nonlinear RGB, constructed as a weighted sum of the RGB values, and two color difference values $\mathrm{Cr}$ and $\mathrm{Cb}$ that are formed by subtracting luma from RGB red and blue components.

$$
\begin{aligned}
& \mathrm{Y}=0.299 \mathrm{R}+0.587 \mathrm{G}+0.114 \mathrm{~B} \\
& \mathrm{Cr}=\mathrm{R}-\mathrm{Y} \\
& \mathrm{Cb}=\mathrm{B}-\mathrm{Y}
\end{aligned}
$$

This color space is suitable for skin color modeling since it is simple to convert from $\mathrm{RGB}$ to $\mathrm{YCrCb}$ [9].

Face image segmentation using skin color model is done by the following steps:

1) Read Original Image.

2) Convert image to $\mathrm{YCrCb}$ color space

3) Apply proper threshold to detect skin region in image. Figure 2 shows the skin region detected.

4) Apply contour detection.

The resulting image was then used to detect the boundaries in the face. The function retrieves contours from the binary image and returns the number of retrieved contours. Figure 3 shows the result of boundary detection where the face, the eyes and the nose boundaries can be clearly seen. The largest boundary represents the boundary of the face image and was considered for further processing.

\subsection{Algorithm}

In this work, two algorithms have been studied, namely the Principal Components Analysis (PCA), as described by Turk and Pentland [10] and secondly the Chain Codes.

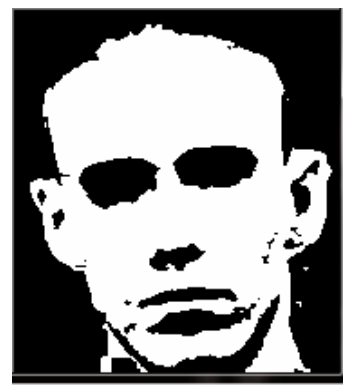

Figure 2. Result of skin detection.

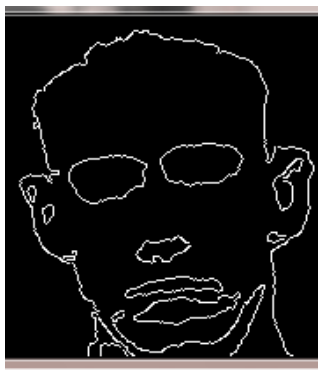

Figure 3. Result of Contour detection.

\subsubsection{The PCA Procedure}

Given an s-dimensional vector representation of each face in a training set of $\mathrm{M}$ images, PCA tends to find a t-dimensional subspace whose basis vectors correspond to the maximum variance direction in the original image space. This new subspace is normally low dimensional. New basis vectors define a subspace of face images called face space. All images of known faces are projected onto the face space to find sets of weights that describe the contribution of each vector. To identify an unknown image, that image is projected onto the face space as well to obtain its set of weights. By comparing a set of weights for the unknown face to sets of weights of known faces, the face can be identified.

The main steps of PCA Algorithm are:

a) Determine PCA subspace from training data. $\mathrm{i}^{\text {th }} \mathrm{im}$ age vector containing $\mathrm{N}$ pixels is in the form

$$
x^{4}-[x, \cdots, x]
$$

b) Store all $p$ images in the image matrix

$$
\mathrm{X}=\left[\mathrm{x}^{2}, \mathrm{n}_{2}, \mathrm{x}^{\mathrm{P}}\right]
$$

c) Compute covariance matrix

$$
\Omega=\mathrm{xx}^{2}
$$

d) Compute eigenvalues and eigenvectors

$$
\Omega V=\Delta V
$$

where, $V$ is the vector of eigenvalues of the covariance 
matrix.

e) Order eigenvectors

$$
\mathrm{V}=\left[\mathrm{v}_{2}, \mathrm{v}_{2}, \cdots=\mathrm{v}_{\mathrm{P}}\right]
$$

f) Order the eigenvectors in $V$ according to their corresponding eigenvalues in descending order. Keep only the eigenvectors associated with non-zero eigenvalues. This matrix of eigenvectors forms the eigenspace $v$, where each column of $V$ is the eigenvector. Visualized eigenvectors of the covariance matrix are called eigenfaces.

\subsubsection{Chain Codes}

Chain code is an object method representation based on its boundary. The chain code of a boundary is determined by specifying a starting pixel and the sequence of unit vectors obtained from going either left, right, up, or down in moving from pixel to pixel along the boundary. The advantage of using chain codes is that it preserves information and allows considerable data reduction. The first approach for representing digital curves using chain code was introduced by Freeman in 1961[11], and it is known as Freeman Chain Code (FCC).

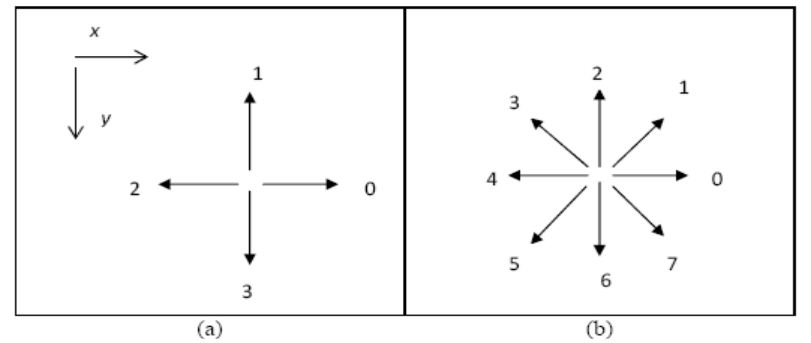

Figure 4. (a) 4 directional chain codes, (b) 8-directional chain codes.

This code follows the contour in counter clockwise manner and keeps track of the directions as we go from one contour pixel to the next. This representation is based on 4-connectivity or 8- connectivity of the segments [12]. The direction of each segment is coded by using a numbering scheme as shown in Figure 4(a) and Figure 4(b).

The first difference of a chain code is the number of direction changes (in counterclockwise) between 2 adjacent elements of the code. This method is invariant to rotation. Figure 5 shows the eight directions that a number in a chain code can follow.

The number of direction changes for each pair of pixels in the contour is determined. The feature vector is created by counting the direction changes of each face's contours.

\subsection{Database}

The study used the Essex Face Database [13]. The train- ing set included 400 images for 20 persons (20 images per person). Sample faces are shown in Figure 1. Face images were of $180 \times 200$ pixels. There were small variations in facial expression, luminance, scale and viewing angle and were shot at different time. Limited side movement and tilt of the head were tolerated.

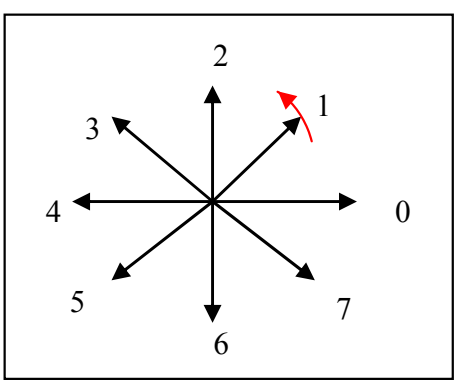

Figure 5. First Difference.

\section{Results}

Experiments done included testing chain codes with images on which canny edge detection and contour algorithm were performed. Secondly, testing chain codes by using the proposed skin color model followed by contour detection was done. Both results were compared with PCA algorithm, as shown in Table 1. The corresponding False Acceptance Rate (FAR) and False Rejection Rate (FRR) are also determined.

Table 1. Face Recgnition Results.

\begin{tabular}{|c|c|c|c|}
\hline & $\begin{array}{c}\text { Recognition } \\
\text { Rate }\end{array}$ & FAR & FRR \\
\hline PCA & $87.5 \%$ & 0.8 & 0.2 \\
\hline $\begin{array}{c}\text { Chain codes using canny } \\
\text { edge detection and contour } \\
\text { detection }\end{array}$ & $70 \%$ & 0.4 & 0.1 \\
\hline $\begin{array}{c}\text { Chain Codes using Skin } \\
\text { Model and contour detection }\end{array}$ & $95 \%$ & 0.2 & 0.1 \\
\hline
\end{tabular}

As shown in Table 1, the proposed system yields a higher recognition rate when using the skin color model, than when using canny edge detector. PCA outperforms chain codes when the latter uses the canny edge detection. This can be explained by the fact that the canny edges contains a lot of noise which impacts performance,

This study shows that chain codes can be used as features for face recognition. Further works include testing this method for ear recognition and ultimately creating a multi-model face and ear recognition system.

\section{REFERENCES}

[1] W. W., Bledsoe, "The Model Method in Facial Recognition," Technical Report, PRI 15, Panoramic Research, Inc., Palo Alto, California, 1964.

[2] W. Zhao, R. Chellappa, J. Phillips and A. Rosenfeld, 
"Face Recognition in Still and Video Images: A Literature Survey," ACM Computing Surveys, Vol. 35, 2003, pp. 399-458. doi; $10.1145 / 954339.954342$

[3] M. Seul, O. Lawrence and M. J., Sammon, "Practical Algorithms for Image Analysis: Description," Examples and Code, edisi kebrp. USA: Cambridge University Press, 2000.

[4] A. McAndrew, "Introduction to Digital Image Processing with Matlab," USA: Thomson Course Technology: pp. 353, 2004.

[5] C. Jiang, Y. Zhao, W. Xu and X. Meng, "Research of Fingerprint Recognition," 2009 Eighth IEEE International Conference on Dependable, Autonomic and Secure Computing, 2009, pp. 847-848. doi:10.1109/DASC.2009.102

[6] P. Kakumanu, S. Makrogiannis and N. Bourbakis, "A Survey of Skin-ColorModeling and Detection Methods", Pattern Recognition 40, 2007, pp 1106- 1122. doi:10.1016/j.patcog.2006.06.010

[7] V. Vezhnevets, V. Sazonov and A. Andreeva, "A Survey on Pixelbased Skin Color Detection Techniques," In
GraphiCon, Moscow, Russia, 2003.

[8] R. Gonzalez and E. Woods, Digital Image Processing, 2nd edition, Prentice Hall, 2002.

[9] S. L. Phung, A. Bouzerdoum and D. Chai, "A Novel Skin Color Model in Ycbcr Color Space and Its Application to Human Face Detection," IEEE International Conference on Image Processing (ICIP'2002), Vol. 1, 2002, pp. 289-292.

[10] M. Turk and A. Pentland, "Eigenfaces for Recognition," Journal of Cognitive Neuroscience, Vol. 3, 1991, pp. 71-86.doi:10.1162/jocn.1991.3.1.71

[11] H. Freeman, "On the Encoding of Arbitrary Geometric Configurations," in Proceedings of IRE Translation Electron Computer, New York, 1961, pp. 260-268.

[12] R. C. Gonzales and R. E. Woods, Digital Image Processing, 2nd Ed. Upper Saddle River, N. J.: Prentice-Hall, Inc., 2002.

[13] Spacek L., Computer Vision Science Research Projects, 2007, Retrieved on July 20, 2012 from Essex University website: http://cswww.essex.ac.uk/mv/allfaces/faces94.html. 